\section{Mucosa associated lymphoma of the lung}

\author{
P H B Bolton-Maggs, A Colman, \\ G R Dixon, M W Myskow, J G Williams, \\ R J Donnelly, C R K Hind
}

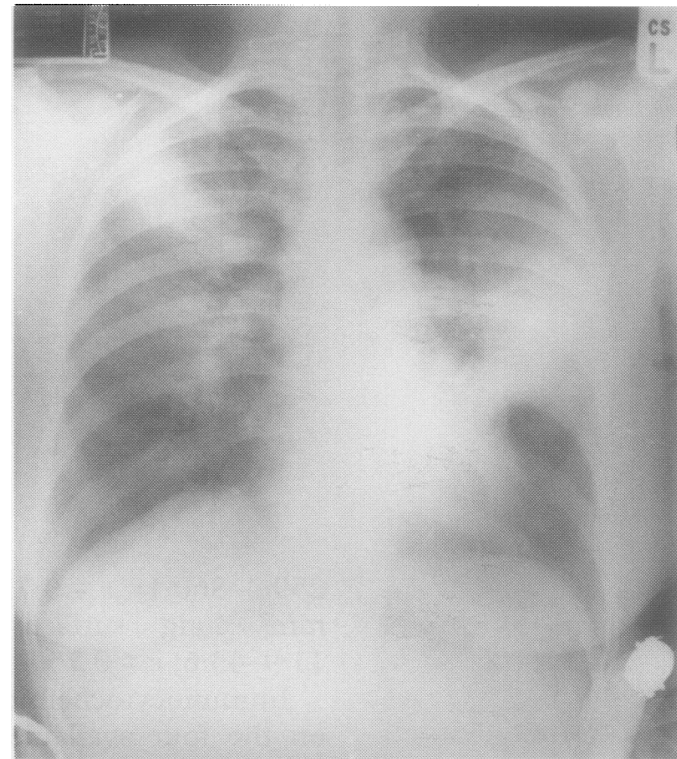

Figure 1 Case 1: Chest radiograph at first presentation showing infiltrates in both lungs.

to normal. Her chest radiograph also returned to normal with the exception of an area of atelectasis in the right mid zone.

She remained asymptomatic for eight months when progressive dyspnoea recurred. On examination crackles were audible at the left lung base and new shadows had appeared on her chest radiograph. An open lung biopsy was performed which revealed macroscopically nodular lungs with normal mediastinal nodes. The histological findings are described below. Computed tomographic (CT) scanning of chest and abdomen showed no nodal spread or other foci of disease, but trephine marrow biopsy specimens showed lymphomatous infiltration. Treatment with steroids at an initial dose of $20 \mathrm{mg}$ prednisolone daily and intermittent chlorambucil (4 mg daily for 14 days each month) resulted in a good clinical response within a month. On withdrawal of steroids she had a recurrence of symptoms; these were restarted and she has been well for over three years follow up on chlorambucil and steroids. Her chest radiograph now shows residual scarring only.

CASE 2

A 66 year old woman presented with a two month history of cough, shortness of breath, and weight loss. Examination was unremarkable. Her chest radiograph showed multiple nodular lesions throughout both lung fields (fig 2). The appearances of CT scans were suggestive of areas of consolidation. Bronchoscopic examination and transbronchial biopsy specimens were normal. Percutaneous lung biopsy specimens showed non-specific organising pneumonitis. There was some improvement in symptoms with treatment with Augmentin (amoxycillin and clavulanic acid) and prednisolone over two months. Her condition deteriorated over the subsequent two months, however, with increasing chest radiographic shadowing. At thoracotomy both upper and lower lobes of eosinophilia. A diagnosis of atypical pneumonia was made, although all cultures and serological tests were negative, and no circulating autoantibodies were subsequently found. Her symptoms resolved spontaneously over two months and her lung function tests returned 
Figure 2 Case 2: Chest radiograph at presentation, showing nodular

shadowing in both lungs.

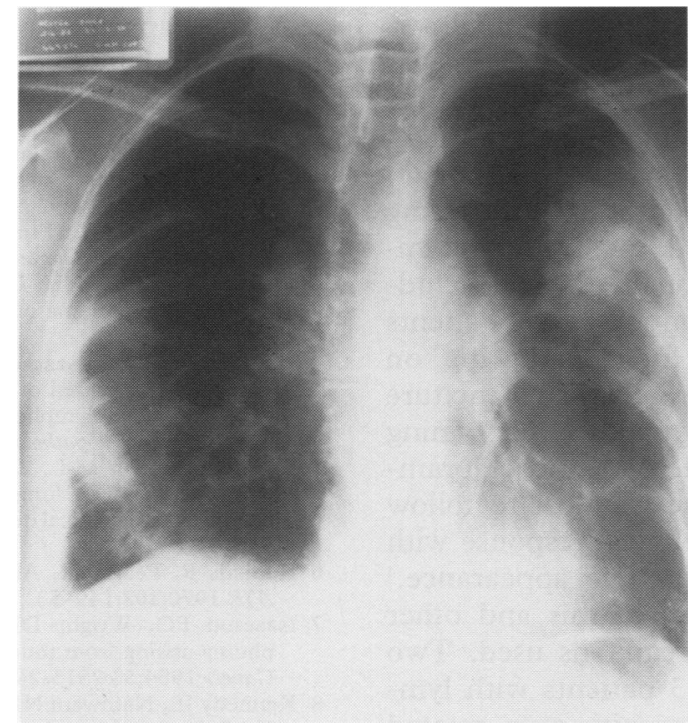

the left lung were studded with firm nodules varying in size from a few millimetres to several centimetres. CT scanning of chest and abdomen showed no evidence of nodal spread or other foci of disease. Treatment with monthly pulses of prednisolone, $40 \mathrm{mg}$ daily for 5 days, and chlorambucil, $4 \mathrm{mg}$ daily for 14 days, was followed by resolution of symptoms and signs and she remains symptom free four years later on intermittent chlorambucil alone. Her chest radiograph now shows residual scarring only.

\section{HISTOLOGY}

The histological features were similar in both cases. The pulmonary architecture was effaced by a dense interstitial lymphoid infiltrate containing occasional germinal centres. Cytologically this was characterised by an admixture of cells, the most prominent of which was a small centrocyte like cell with an angulated or cleaved nucleus and often a small nucleolus. Small numbers of larger transformed cells with ovoid vesicular nuclei with prominent nucleoli were also present (fig 3A). Also noted were varying numbers of plasma cells, eosinophils, and macrophages. There was infiltration of the bronchial epithelium by centrocyte like cells forming characteristic lymphoepithelial lesions (fig 3B). In case 2 occasional small granulomas were seen. Immunohistochemical staining revealed the lymphoid cells to be B cells; staining for immunoglobulin light chains showed kappa light chain restriction indicating a monoclonal proliferation. These appearances were interpreted as being indicative of primary low grade lymphoma (MALT lymphoma) of the lung. ${ }^{5}$

\section{Discussion}

The term "pseudolymphoma" of the lung was adopted by Saltzstein ${ }^{1}$ in 1963 to describe localised tumours of small lymphocytes, which were more indolent than those at other sites, with a long latency before spread and therefore a long survival of affected patients. In addition to small lymphocytes, these tumours characteristically contain a mixed cellular infiltrate and true germinal centres, while local lymph nodes are not involved. "Pseudolymphoma" was thought to be a chronic inflammatory process, perhaps a manifestation of autoimmunity. ${ }^{16}$ Several recent studies of "pseudolymphomas" and more diffuse lymphoid proliferations within the lung (including many cases of lymphocytic interstitial pneumonitis) with immunohistochemical techniques, however, have indicated monoclonality of the lymphoid population in most cases. ${ }^{3479}$ The majority are B cell in origin and the stomach is the commonest site. "Pseudolymphoma" is thus a misnomer, and such tumours and many cases of lymphocytic interstitial pneumonia should be recognised as true lymphomas of MALT, with histological features distinct from nodal lymphomas.

As MALT lymphoma is uncommon there are no clear guidelines for its management. ${ }^{1-12}$ Localised disease has been successfully treated by excision alone. ${ }^{15}$ Patients with more diffuse disease within the lungs tend to have symptoms and have a worse prognosis. ${ }^{59}$ Numerous different chemotherapeutic agents have been tried for patients with more diffuse disease, either alone or in combination, with varying success. ${ }^{348}$ One 48 year old woman with a recurrent pleural effusion associated with lymphoid interstitial pneumonitis (monoclonality of lymphocytes found) responded partially to unacceptably high doses of
Figure 3 (A) Close up view of lymphoid infiltrate showing an admixture of centrocyte like cells and transformed cells

(haematoxylin and eosin). (B) Bronchial epithelium infiltrated by lymphoid cells giving rise to

lymphoepithelial lesions

(haematoxylin and eosin).

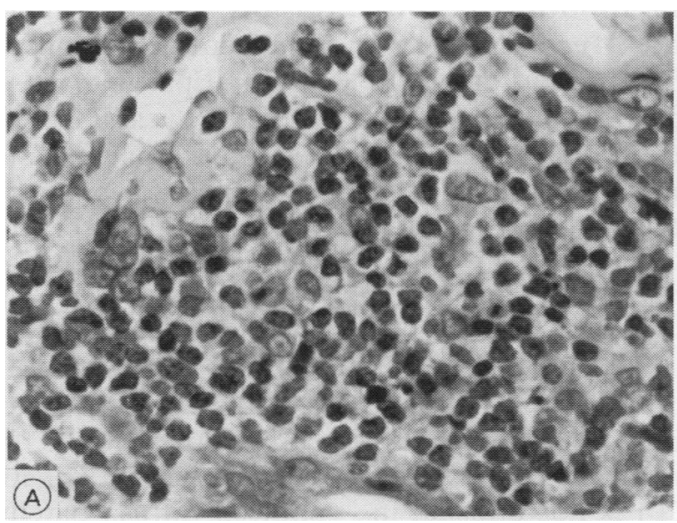


steroids and the addition of chlorambucil permitted withdrawal of steroids. She was treated with chlorambucil for a year and was symptom free on no treatment at the time of reporting. ${ }^{3}$ A review of 15 cases of pulmonary lymphoproliferative conditions including three originally defined as "pseudolymphoma" showed monoclonality in 13 (including all three pseudolymphomas). Patients with diffuse or patchy lung shadowing on radiography had been treated with a mixture of chemotherapy regimens containing steroids, two receiving steroids and chlorambucil alone. One of these was lost to follow up and the other had a clinical response with no change in the radiographic appearance. ${ }^{4}$ Insufficient detail is given in this and other reports ${ }^{8}$ to evaluate the regimens used. Two of a reported series of 13 patients with lymphocytic interstitial pneumonitis were treated with chlorambucil and steroids with improvement in one. ${ }^{10}$ There is no consensus established for the treatment of these conditions. As histologically these patients have low grade lymphoma, we chose to treat our two patients with steroids and chlorambucil, agents well tried and effective for other types of low grade B cell lymphoma and chronic lymphatic leukaemia and used successfully in some patients with "pseudolymphoma". To date this approach has produced a good response in these two patients.

1 Saltzstein SL. Pulmonary malignant lymphomas and pseudolymphomas: classification, therapy and prognosis. Cancer 1963;16:928-55.

2 Gibbs AR, Seal ME. Primary lymphoproliferative conditions of the lung. Thorax 1978;33:140-52.

3 Herbert A, Wright DH, Isaacson PG, Smith JL. Primary malignant lymphoma of the lung. Hum Pathol 1984;15: 415-22.

4 Addis BJ, Hyjek E, Isaacson PG. Primary pulmonary lymphoma: a reappraisal of its histogenesis and its relationship to pseudolymphoma and lymphoid interstitial pneumonia. Histopathology 1988;13:1-17.

5 Li G, Hansmann M-L, Zwingers T, Lennert K. Primary lymphomas of the lung: morphological, immunohistochemical and clinical features. Histopathology 1990;16: 519-31.

6 Robbins R, Peale AR, Al-Saleem T. Pseudolymphomas. AFR 1970;108:149-53.

7 Isaacson PG, Wright DH. Extranodal malignant lymphoma arising from mucosa-associated lymphoid tissue. Cancer 1984;53:2515-24.

8 Kennedy JL, Nathwani NB, Burke JS, Hill RL, Rappaport H. Pulmonary lymphomas and other pulmonary lymphoid lesions. Cancer 1985;56:539-52.

9 Herbert A, Walters MT, Cawley MID, Godrey RC. Lymphocytic interstitial pneumonia identified as lymphoma of mucosa associated lymphoid tissue. $f$ Pathol 1985;146:129-38.

10 Strimlan CV, Rosenow EC, Weiland LH, Brown LR. Lymphocytic interstitial pneumonitis. Review of 13 cases. Ann Intern Med 1978;88:616-21.

11 Goldman JM. The lymphomas. In: Hoffbrand AV, Lewis SM, eds. Postgraduate haematology. 3rd edn. Oxford: Heinemann, 1989:502-29.

12 Krikorian JG, Portlock CS, Cooney DP, Rosenberg SA. Spontaneous regression of non-Hodgkin's lymphoma: a report of nine cases. Cancer 1980;46:2093-9.
Culture of

\section{Mycobacterium kansasii} in the blood of an HIV negative patient

\author{
D Veale, D Fishwick, J E S White, \\ A D Gascoigne, K Gould, P A Corris
}

\begin{abstract}
A 23 year old man with a congenital myelodysplastic disorder and fibrosing lung disease received treatment with prednisolone. After nine months his condition deteriorated and Mycobacterium kansasii was isolated from blood cultures and lymph node biopsy specimens. He responded to antituberculous treatment. $M$ kansasii has not previously been isolated from the blood stream of HIV negative patients.
\end{abstract}

(Thorax 1993;48:672-673)

Mycobacterial bacteraemia with organisms other than $M$ tuberculosis (mycobacteria other than tuberculosis, MOTT) is not unusual in patients with acquired immunodeficiency syndrome (AIDS), ${ }^{1}$ and the $M$ avium complex is the most frequently isolated subtype. ${ }^{2}$ There are few reports of isolation of nontuberculous mycobacteria from blood cultures in non-HIV patients. ${ }^{3}$ We report a young man with a familial myelodysplasia and persistent Epstein-Barr virus infection who developed widespread $M$ kansasii infection with isolation of the organism from blood cultures.

\section{Case report}

A 23 year old man developed fever and rigours five weeks after returning from a holiday in Spain. He then developed a cough with clear sputum and nausea. In his past history he had suffered severe varicella and recurrent herpes simplex infections and a persistent Epstein-Barr virus infection from the age of 20 years. An older brother had died of refractory anaemia with excess lymphoblasts. Genetic studies had determined familial dysplasia with constitutional inversion of chromosome $1 .^{4}$ Immunological studies had shown leucopenia with a profound lymphopenia but no other abnormality. On examination he had gross digital clubbing and generalised lung crackles. White blood cell count (WBC) was $0.9 \times 10^{9} / 1$ and he was HIV antibody negative. Chest radiography showed diffuse patchy parenchymal shadow- 